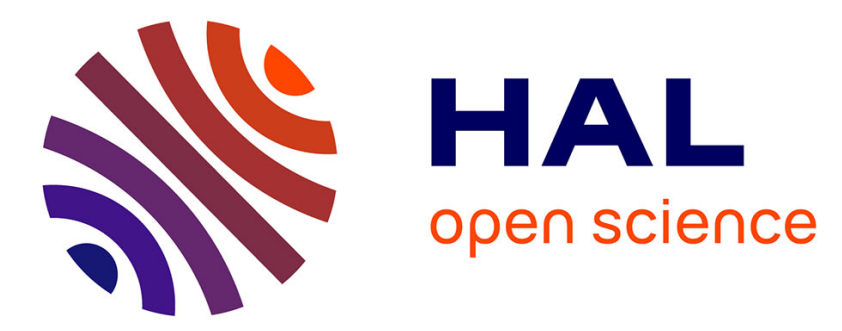

\title{
Réflexion sur l'institutionnalisation récente des memory studies
}

\author{
Sarah Gensburger
}

\section{To cite this version:}

Sarah Gensburger. Réflexion sur l'institutionnalisation récente des memory studies. Revue de Synthèse, 2011, 132-VI (3), pp.1-23. halshs-00926329

\section{HAL Id: halshs-00926329 \\ https://shs.hal.science/halshs-00926329}

Submitted on 14 Oct 2014

HAL is a multi-disciplinary open access archive for the deposit and dissemination of scientific research documents, whether they are published or not. The documents may come from teaching and research institutions in France or abroad, or from public or private research centers.
L'archive ouverte pluridisciplinaire HAL, est destinée au dépôt et à la diffusion de documents scientifiques de niveau recherche, publiés ou non, émanant des établissements d'enseignement et de recherche français ou étrangers, des laboratoires publics ou privés. 
"Réflexion sur l'institutionnalisation récente des memory studies", rubrique Chroniques de la Recherche, Revue de Synthèse (Springer) tome 132, 6e série, $n^{\circ}$ 3, 2011, p. 1-23.

\section{Chronique de la recherche}

\section{Regard sociologique sur l'institutionnalisation actuelle des memory studies}

A partir du milieu des années 80 , un nombre croissant de chercheurs en sciences sociales se sont intéressés à la «mémoire » pour renouveler des approches disciplinaires dont la valeur heuristique leur semblait en voie d'épuisement. L'anthropologie y a vu un moyen de contourner les apories de la notion de «culture ${ }^{1}$, 1 'histoire une manière de renouveler l'étude tant des «mentalités » que de l'«événement $»^{2}$. Vingt ans plus tard, ce qui a depuis pris l'ampleur d'un « memory boom » fait à son tour l'objet de nombreuses critiques ${ }^{3}$. Cellesci partent notamment du constat de la relative impuissance des analyses existantes à expliquer l'inflation des questions mémorielles - à laquelle elles ont d'ailleurs largement contribué pour poser ouvertement la question de la cumulativité de recherches désormais si nombreuses qu'il est matériellement impossible pour un seul chercheur d'en prendre intégralement connaissance.

Depuis peu, conscients de l'impasse dans laquelle se trouvent effectivement les travaux qui prennent la «mémoire » pour objet, plusieurs chercheurs oeuvrent à la mise en place d'un champ de recherches intégré ${ }^{4}$, voire d'une nouvelle discipline ${ }^{5}$ : les «memory studies ». Celles-ci comptent désormais des centres de recherche ${ }^{6}$, des lieux d'échange académique ${ }^{7}$,

\footnotetext{
${ }^{1}$ GOODY, 1977 ; ZONABEND, 1980.

${ }^{2}$ NORA, 1978.

3 Berliner, 2005; Winter, 2000 et 2006. Presque exactement contemporains, les textes respectivement, historien et sociologue, CONFINO, 1997 ; OLICK et ROBBINS, 1998, constituent la première formulation d'un bilan, encore enthousiaste, des travaux sur la mémoire qui se multiplient alors depuis quinze ans.

${ }^{4}$ BROWN et al., 2009 ; OLICK, 2009. La première Memory conference organisée par la New School for Social Research (New York) en février 2008, s’intitulait « Is an Interdisciplinary Field of Memory Studies Possible ?». ${ }^{5}$ ROEDIGER et WERTSCH, 2008.

${ }^{6}$ Comme, par exemple, le Center for Interdisciplinary Memory research (Essen, Allemagne), le Center for the Study of Cultural Memory (Londres, Grande-Bretagne), le Center for Popular Memory (Cape Town, Afrique du Sud), le Center for Public Memory and Ethnic Studies du Lewis and Clark College (Portland, USA), le Mercator Research Group on Structure of Memory de la Ruhr Universität (Borhum, Allemagne), le Center for Research in Memory, Narrative and Histories de l'Université de Brighton (Royaume-Uni) ou le Warwick Center for Memory studies (Grande-Bretagne), même si dans ce dernier cas, le départ d'Andrew Hoskins pour l'Université de Nottingham semble avoir mis en sommeil ses activités. Un «Memory Studies Project» est également en gestation à McGill University (Canada).

${ }^{7}$ Le site d'informations et de réseaux académiques H-Net abrite depuis 2009 une liste H-Memory dont les créateurs entendent proposer un forum internet pour les «scholars in memory studies ». Depuis la première conférence, en 2008, la New School for Social Sciences organise désormais annuellement une «Memory Conference » qui a pour objectif de permettre aux chercheurs en memory studies de se retrouver collectivement.
} 
une revue ${ }^{8}$ et une collection éditoriale $e^{9}$ éponymes. Elles donnent lieu à des enseignements ${ }^{10}$ et à la publication de manuels ${ }^{11}$ et de recueils de textes ${ }^{12}$.

Dans le discours de ses protagonistes, l'institutionnalisation des memory studies est censée mettre un terme à la «non-paradigmatic, transdisciplinary, centerless condition» des nombreuses études qui portent pourtant toutes, bel et bien, sur la «mémoire $»^{13}$. Elle doit favoriser l'émergence d'un arsenal théorique et méthodologique spécifique et transdisciplinaire adapté au traitement d'un objet pensé comme singulier, arsenal qui est luimême considéré comme indispensable à une production cumulative de connaissances en la matière ${ }^{14}$. La constitution de ce champ de recherches dédié doit ainsi permettre de dépasser plusieurs des impasses dans lesquelles se trouvent aujourd'hui les travaux issus du «memory boom ». Nombreuses, ces impasses touchent à deux questions centrales, elles-mêmes liées : l'obstacle de la «réification» et l'énigme de la «réception». Les (nombreux) travaux existants n'auraient, tout d'abord, pas réussi à comprendre de manière non-réifiante et opératoire l'articulation entre mémoire individuelle et mémoire collective autrement que par l'utilisation métaphorique de termes psychanalytiques ${ }^{15}$. La synergie attendue des memory studies doit, ensuite, permettre de résoudre la question dite de la «réception » par les individus $^{16}$ d'une mémoire pensée comme produite par des «acteurs» ou autres « entrepreneurs » de mémoire ${ }^{17}$.

\footnotetext{
${ }^{8}$ Depuis janvier 2008, les éditions SAGE publient une nouvelle revue scientifique Memory Studies. Pour ce qui est du champ francophone, la création en 2006 de la revue franco-québecoise Conserveries mémorielles participe du même processus de disciplinarisation. Cette revue a vocation à être un «lieu de diffusion des travaux du domaine des sciences humaines et sociales portant sur des problématiques liées à la mémoire ».

${ }^{9}$ Une collection «Memory Studies » existe désormais chez la maison d'édition Palgrave MacMillan, elle est dirigée par Andrew Hoskins (par ailleurs rédacteur en chef de la revue éponyme) et John Sutton (voir note suivante). De même la collection «Memory and Narrative» dirigée par Susanne Radstone chez Transaction Publishers.

${ }^{10}$ Il ne s'agit pas ici de dresser un inventaire de ces enseignements, pour un exemple, celui intitulé «Social Memory» dispensé par John Sutton à Macquarie University (Sydney, Australie). Il existe aussi des undergraduate program dédiés comme le Luce Program in Individual and Collective Memory, Washington University (St Louis, USA).

${ }^{11}$ ERLl et NUNNING, 2008 ; WoOdS et ByATt, 2008 ; BROCKMEIER, 2010; GUdehUS et al., 2010a ; RADSTONE, 2010.

${ }^{12}$ Rossington et WhiteheAd, 2007 ; LeVy et al., 2011.

${ }^{13}$ OLICK, 2008b, p. 27. Il n'est bien sûr pas question ici de nier les divergences entre, et hésitations de, celles et ceux qui participent, à des degrés divers, de l'institutionnalisation de ce nouveau champ de recherches, par exemple SUTTON, 2009 ; RADSTONE, 2008. Il s'agit par contre de défendre une position plus radicale à l'égard de cette évolution.

${ }^{14}$ ROEDIGER et WERTSCH, 2008.

${ }^{15}$ Crane, 1997 ; Klein, 2000.

16 KANSTEINER, 2002.

${ }^{17}$ Schwartz et SCHUMAN, 2005 ; ARMSTRONG et Crage, 2006.
} 
Or, et s'ils sont majoritairement anglo-saxons, les chercheurs qui participent de cette institutionnalisation des memory studies s'inscrivent dans une généalogie intellectuelle dans laquelle Maurice Halbwachs est présenté comme le père fondateur. Bien que tous ne s'accordent pas sur le sens à donner au terme halbwachsien de « mémoire collective " ${ }^{18}$, ils y font référence comme à une notion canonique pour leur discipline. Dans les faits, aucun de ces travaux ne mobilise toutefois empiriquement la grille d'analyse proposée par Halbwachs dans sa «théorie sociologique de la mémoire ${ }^{19}$. Il est pourtant possible de s'appuyer effectivement, et de manière empiriquement opératoire, sur l'œuvre d'Halbwachs pour défendre une autre modalité de renouvellement des travaux sur la mémoire. Cet article entend plaider la cause de cette voie alternative pour considérer que la « mémoire » n'est pas un objet de recherche spécifique mais doit être étudiée au sein et à l'aide des outils et méthodes ordinaires des disciplines, au premier rang desquelles la sociologie.

Cet argument sera développé ici en confrontant la théorie élaborée par Halbwachs et l'usage qui en est fait dans la plupart des travaux contemporains qui se regroupent aujourd'hui sous la bannière des memory studies. Il ne s'agit pas de revendiquer la connaissance du «véritable » Halbwachs mais plutôt de montrer en quoi l'articulation entre sa théorie et les questionnements contemporains peut effectivement offrir un cadre opératoire pour la conduite de travaux empiriques novateurs et susceptibles de participer au renouvellement des études de mémoire collective et, ce faisant, d'abord à la connaissance du social et de ses dynamiques en général.

\section{Les memory studies : penser la mémoire à l'aide de la théorie halbwachsienne ?}

«Since its emergence as a distinct field of study as a result of the pioneering work of Halbwachs, scholars in recent decades have demonstrated an enormous interest in collective memory $»^{20}$. Ecrite par plusieurs des artisans de l'institutionnalisation en cours des memory studies, cette phrase résume parfaitement le statut de « field founder » donné à Halbwachs ${ }^{21}$. Qualitativement, c'est en ces termes qu'il est présenté dans la plupart des articles réunis, depuis 2008, dans la revue Memory Studies et censés marquer l'acte de naissance du champ éponyme. Quantitativement, une requête par mots clef sur les moteurs de recherche Google et

\footnotetext{
${ }^{18}$ KANSTEINER, 2002 ; OLICK, 2007.

${ }^{19}$ HALBWACHS, 1994, p. VIII.

${ }^{20}$ BROWN et al., 2009, p. 119.

${ }^{21}$ OLICK, 2009.
} 
Google scholar montre qu'Halbwachs est l'auteur le plus souvent cité dès lors qu'il est question de « memory studies $»^{22}$.

Cette filiation proclamée met en avant la notion de « mémoire collective » comme le principal héritage du sociologue français : «Stemming from Halbwachs's seminal book in 1925, the concept of collective memory has changed the way in which researchers in both the social sciences and the humanities understand what it means for individuals and groups to remember $\gg{ }^{23}$.

En résumé, «Maurice Halbwachs founded the field of collective memory ${ }^{24}$ qui regroupe aujourd'hui des $«$ scholars of collective memory ${ }^{25}$ qui placent leurs travaux sous le « label collective memory $»^{26}$.

Omniprésente aujourd'hui dans l'institutionnalisation des memory studies comme, jusqu'ici, dans le développement du "memory boom », la référence à l'œuvre halbwachsienne reste cependant de pure forme. Elle est le plus souvent cantonnée à l'introduction générale du propos. Aucun des travaux qui se réclament de cette généalogie ni ne cite la prose du sociologue français ni ne la mobilise effectivement pour conduire des recherches empiriques. Ce formalisme de l'héritage halbwachsien se retrouve dans les différents paysages nationaux et l'ensemble des disciplines concernées. Alors même qu'il le reconnaît comme le père fondateur des memory studies dont il est lui l'un des principaux protagonistes, J.K. Olick constate lui-même que si Halbwachs est « universally cited, very often such cites seem more totemic thant substantive or engaged ». Il considère simultanément que le terme de «mémoire collective », censé incarné l'ensemble de la théorie d'Halbwachs sur la mémoire, « is not an operational concept: There seems to be very little agreement on what it is - if indeed it is anything - or on what sort of a metric would apply $»^{27}$. En fait, si la pensée halbwachsienne et la notion de «mémoire collective » fédèrent les divers auteurs qui participent aujourd'hui de l'institutionnalisation des memory studies, c'est en quelque sorte au second degré. Elles constituent des références communes qu'il convient d'invoquer mais aussi de critiquer.

\footnotetext{
${ }^{22}$ Dans une première étape, la lecture des articles et livres scientifiques sur les «memory studies » produits depuis 2007 nous a permis de dresser une liste des auteurs régulièrement cités. Ensuite, des recherches croisées ont été effectuées. Pour le détail des résultats voir les tableaux regroupés en annexe. Pour une recherche plus générale sur la diffusion scientifique et profane du terme de «mémoire collective » lui-même, BRIAN, 2008.

${ }^{23}$ FivUSH, 2010, p. 78.

${ }^{24}$ SCHWARTZ et SCHUMAN, 2005, p. 183.

${ }^{25}$ SPILlman, 1998.

${ }^{26}$ OLICK, 2008a, p. 9 ; HUTTON, 2010. C'est d'ailleurs le terme de « Collective memory » que J.K. Olick et ses deux co-auteurs ont choisi pour donner son titre à leur reader, LEVY et al., 2011, et ce malgré les critiques qu'ils ont eux-mêmes formulées, à plusieurs reprises, à l'égard du concept halbwachsien, OLICK, 1999b ; VINITZKYSEROUSSI, 2002.

${ }^{27}$ Olick, 2008a, respectivement p. 26 et 85.
} 
Ces critiques portent sur deux points principaux qui font apparaître, en creux, la lecture dominante qui est faite de la théorie halbwachsienne dans la plupart des études de mémoire collective aujourd'hui regroupées sous la bannière des memory studies. Le premier concerne le privilège absolu qu'Halbwachs accorderait au "présent» sur le "passé ». Le second touche à la réification de la «mémoire collective» et à la conception transcendantale de la société qu'induirait cette notion.

Ce qui est désigné le plus souvent comme le «'presentist' argument about collective memory $»^{28}$ est, en effet, largement présenté comme le principal apport de la pensée d'Halbwachs. Lewis Coser, traducteur et l'un spécialistes anglophones du sociologue français, axe ainsi sur cette dimension la synthèse qu'il lui consacre :

« Halbwachs was without doubt the first sociologist to stress that our conceptions of the past are affected by the mental images we employ to solve present problems, so that collective memory is ensentially a reconstruction of the past in the light of the present $»^{29}$.

Cependant, et tout en reconnaissant l'importance de l'intuition d'Halbwachs, qu'il le conduit à la prise en compte d'un « contexte social ${ }^{30}$ au statut heuristique jamais vraiment spécifié,

L. Coser formule sa principale critique précisément sur cette question :

«If the presentist approach were pushed to its ultimate consequences, it would suggest that there is no continuity in history altogether. It would make history a series of snapshots taken at various times and expressing various perspectives $»^{31}$.

Ici formulé sur un plan théorique, ce reproche de l'absence de prise en compte de la « continuité historique »s'appuie ailleurs sur des études de $\operatorname{cas}^{32}$. Dans ce cadre, la notion de path-dependence, empruntée à la science politique contemporaine, est régulièrement mobilisée pour amender l'approche d'Halbwachs. Elle est présentée comme «a crucial innovation that corrects the strong presentist bias in previous work on collective memory (Halbwachs 1992). It is now widely accepted that the present interests of social groups cannot

\footnotetext{
${ }^{28}$ OLICK, 2007, p. 42.

${ }^{29}$ COSER, 1992, p. 372.

${ }^{30}$ De SCHWARTZ, 1982 à VINITZKY-SEROUSSI, 2002, le « social context of collective memory » est présenté comme le mécanisme censé expliquer le poids du présent: « an individual memory depends on the social context to which we appeal», HUTTON, 1993, p. 79. Ce point mériterait un article spécifique. Nous y reviendrons brièvement dans la troisième partie de cet article.

${ }^{31}$ COSER, 1992, p. 370.

${ }^{32}$ SChWARTZ, 1982 ; SChWARTZ, 1991 ; SPILlman, 1998 ; Olick, 1999a.
} 
dictate reconstruction of collective memory, for collective memory can only be reshaped within limits that are dependent on the past of commemoration $»^{33}$.

C'est dans ce premier axe de discussion critique de l'héritage d'Halbwachs que trouvent leur origine les nombreux développements qui lui attribuent la paternité de la fameuse distinction entre «Histoire et Mémoire», distinction qui occupe une large part de la littérature du « memory boom » depuis plus de vingt ans. « Embedded in a positivist tradition that sought to uncover universal social laws, Halbwachs enforced the separation of history and memory as modes of knowledge and compartmentalized their functions $»^{34}$. Là encore, et de manière totalement imbriquée avec la question de l'approche présentiste, la proposition théorique d'Halbwachs ne serait pas opératoire. En 1982, et alors même qu'il indiquait le sociologue français comme l'une de ses principales sources d'inspiration, Barry Schwartz concluait déjà son étude de mémoire collective en ces termes :

«While the results of this study come down on the side of a theory which attributes the importance of social origins to the context in which they are recalled, they do not permit us to go as far as Maurice Halbwachs in denying the objectivity of history.[...] Given the constraints of recorded history, the past cannot be literally constructed; it can only be selectively exploited. Moreover, the basis of the exploitation cannot be arbitrary ${ }^{35}$.

La seconde dimension censée caractériser la théorie d'Halbwachs, et qui fait donc simultanément l'objet de commentaires, met en avant non plus le «presentisme» et l'importance du « contexte social » mais la nature « collective » de la mémoire, cette mémoire $\mathrm{du}$ « groupe ». «In describing our investigation as one dealing with « collective memories » we make use of a term advanced by Halbwachs to describe memories of a shared past that are retained by members of a group, large or small, that experienced it $»^{36}$.

La primauté qu'aurait donnée Halbwachs au « collectif», le « groupe » ou la « société » selon les auteurs, est ainsi au cœur de la critique de son œuvre. Elle prend sa forme la plus radicale chez Noa Gedi et Yigal Elam, régulièrement cités depuis 1996, notamment dans les appels récents à la constitution d'un champ de recherches integré ${ }^{37}$ :

«Halbwachs bases his theory on Durkheim's thesis, according to which 'social phenomena' are both external to and independent of individuals and their mental representations [...].

\footnotetext{
${ }^{33}$ SAITO, 2006, p. 305-306 ; ARMSTRONG et CRAGE, 2006 ; JANSEN, 2007.

${ }^{34}$ FALASCA ZAMPONI, 1998, p. 422.

${ }^{35}$ SCHWARTZ, 1982, p. 396. Nous soulignons.

${ }^{36}$ SCHUMAN ET SCOTT, 1989, p. 361.

${ }^{37}$ KANSTEINER, 2002.
} 
While Durkheim's effort seems to have been concentrated on establishing the autonomy of sociology, Halbwachs' effort, it appears, resulted in the complete obliteration of the individual consciouncess as real and determinant $»^{38}$. A des degrés divers, cet argument est au cœur de la plupart des discussions théoriques existantes sur le terme halbwachsien de «mémoire collective ». Il revient par exemple de manière régulière sous la plume du sociologue J.K. Olick. En 1999, dans un texte qui vise précisément à redéfinir la notion, celuici concluait déjà :

« the problem is that Halbwachs does not present us with an integrated paradigm that identifies the unique structures involved in each of these and shows how they are related though he does provide some useful suggestions on all of these matters. Halbwachs is in this sens still a 'nineteenth century' theorist, one who see individual and collective level problems as problems of different orders $»{ }^{39}$.

Dans la même perspective, et d'ailleurs la même année, Jay Winter et Emmanuel Sivan, eux historiens, qualifient l'individu halbwachsien d'« homo-sociologicus, l'homme de la mémoire socialement déterminée » lui opposant «l'homo-actans » qui agit pour la remémoration et s'engage dans la commémoration, à leurs yeux seul véritable objet légitime ${ }^{40}$.

La référence à Halbwachs comme à un père fondateur pour les memory studies s'accompagne donc d'une critique systématique de sa théorie, le plus souvent résumée à la notion de « mémoire collective ». Porteuse d'une conception prétendument présentiste et réifiante de la mémoire, l'œuvre du sociologue français serait ainsi impuissante à résoudre les apories auxquelles le « memory boom » a abouti.

\section{La théorie halbwachsienne : une sociologie opératoire pour l'étude contemporaine de la mémoire}

Le statut d'Halbwachs dans l'institutionnalisation en cours des memory studies est d'autant plus paradoxal que la «théorie sociologique de la mémoire» forgée par celui-ci offre précisément un arsenal sur lequel prendre appui pour dépasser effectivement, et empiriquement, les obstacles sur lesquels butent aujourd'hui les travaux sur la mémoire.

\footnotetext{
${ }^{38}$ GEDI et ELAM, 1996, p. 36.

${ }^{39}$ OLICK, 1999b, p. 336. Dix ans plus tard, et alors qu'il participe à la disciplinarisation des memory studies, le même auteur renouvelle son jugement sur la nature transcendantale du regard halbwachsien : « although there as a many collective memories as there are groups for Halbwachs, and although Halbwahcs emphazizes that it is only individuals who remember, there remain some echoes of Durkheim's collective representations as phenomena sui generis », OLICK, 2008a, p. 89.

${ }^{40}$ WINTER et SIVAN, 1999.
} 
Comme il a déjà été indiqué, il ne s'agit pas, dans ce qui suit, de présenter l'œuvre d'Halbwachs d'une manière qui serait authentique ${ }^{41}$ mais plutôt de mettre en évidence la nature opératoire de celle-ci pour construire une approche contemporaine de la mémoire. La lecture à la fois présentiste et réifiante de la théorie d'Halbwachs sur la mémoire manifeste en effet une incompréhension de sa tentative de conceptualisation tant du temps que de l'espace.

Son appréhension du temps n'est pas fondée sur une distinction entre présent et passé, qui est elle au cœur du temps vécu ${ }^{42}$. Elle passe par l'élaboration de la notion de temps collectif qui est celui de la conscience de l'individu en tant que celui-ci est un être social. Dès les Cadres sociaux de la mémoire, Halbwachs parle ainsi de «la société originale que chaque individu forme en quelque sorte avec lui-même ${ }^{43}$. Il n'y a donc pas d'origine, de moment initial, de la mémoire autre que celui de la conscience de l'individu social dont l'existence s'inscrit dans la durée. C'est dans cette perspective que doit être comprise cette phrase de la conclusion de la Topographie Légendaire : «Quelque époque qu'on envisage, l'attention se dirige non vers l'origine, les événements premiers qui sont peut-être à l'origine de tout ce développement, mais vers les groupes de fidèles... ${ }^{44}$. La démarche d'Halbwachs est ainsi fondamentalement différente d'un présentisme, forme temporelle d'un constructivisme réducteur. Le souvenir présent à la conscience de l'individu social est tout à la fois reconstruit et reconnu :

«Pour que notre mémoire s'aide de celle des autres, il ne suffit pas que ceux-ci nous apportent leurs témoignages [...] Il ne suffit pas de reconstituer pièce à pièce l'image d'un événement passé pour obtenir un souvenir. Il faut que cette reconstruction s'opère à partir de données ou de notions communes qui se trouvent dans notre esprit aussi bien que dans ceux

\footnotetext{
${ }^{41}$ Depuis quelques années, cette présentation a, par ailleurs, été largement entreprise par plusieurs auteurs à tel point qu'Halbwachs a pu être qualifié de «sociologue retrouvé », JAISSON et BAUDELOT, 2007. Elle concerne l'ensemble de l'œuvre, au-delà des seules publications qui portent explicitement sur la mémoire, BAUDELOT et Establet, 1994 ; Montlibert (ed.), 1997 ; Revue D’histoire Des SCIENCES Humaines, 1999 ; NAMER, 2000 ; Deloye et Haroche (eds.), 2004 ; MARCEl, 2004 ; Brian et JAISSON, 2005a; JAISSON et MARCEL, 2005 ; PEQUignot (ed.), 2007 ; BRIAn, 2010. En Allemagne, un intérêt important s'est également fait jour mais le travail de relecture et de réédition/traduction s'est, dans ce cas, davantage concentré sur les œuvres qui ne concernent pas explicitement la mémoire, EGGER et SCHULTHEIS (eds.), 2001a, 2001b, 2001c, 2001d, 2002 ; EGger, 2003, Wetzel, 2009. Une évolution convergente semble se faire jour en Espagne, MARTINEZ GUTIERREZ, 2008 ; REVISTA ANTHROPOS, 2008.

42 JAISSON, 2008.

${ }^{43}$ HALBWACHS, 1994 [1925], p.139.

${ }^{44}$ HalbWACHS, 2008 [1941], p. 163. C'est à l'aune de cette explicitation de sa démarche qu'il convient de comprendre cette phrase tirée de l'introduction de l'ouvrage, et souvent citée pour illustrer le supposé « présentisme » radical d'Halbwachs, SCHWARTZ, 1991: « Si, comme nous le croyons, la mémoire collective est essentiellement une reconstruction du passé, si elle adapte l'image des faits anciens aux croyances et aux besoins spirituels du présent, la connaissance de ce qui était à l'origine est secondaire, sinon tout à fait inutile, puisque la réalité du passé n'est plus là, comme un modèle immuable auquel il faudrait se conformer », HALBWACHS, 2008 [1941], p. 7.
} 
des autres, parce qu'elles passent sans cesse de ceux-ci à celui-là et réciproquement, ce qui n'est possible que s'ils ont fait partie et continuent à faire partie d'une même société. Ainsi seulement, on peut comprendre qu'un souvenir puisse être à la fois reconnu et reconstruit $\gg{ }^{45}$.

Différente du « strong presentist bias » à laquelle elle est régulièrement réduite, la théorie de la mémoire d'Halbwachs est aussi fondamentalement étrangère à la « réification » dont elle est, à des degrés divers, systématiquement accusée. L'opération de reconnaissance/reconstruction qui donne sa dynamique à la mémoire est en effet une opération de localisation intersubjective. Halbwachs forge le concept fondamental d'espace social qui lui permet de penser la continuité de l'individu comme être social et alors même que le temps de conscience dans lequel se trouve celui-ci est toujours au présent ${ }^{46}$. L'espace social est un système structuré de relations interindividuelles ${ }^{47}$. La localisation mémorielle découle ainsi de la place que l'individu occupe dans un ensemble structuré de relations comme de la modification de ce dernier. Elle s'effectue de manière intersubjective selon un mécanisme de reconnaissance mutuelle entre les différents individus qui se situent dans l'espace social :

«Si la mémoire collective tire sa force et sa durée de ce qu'elle a pour support un ensemble d'hommes, ce sont cependant des individus qui se souviennent, en tant que membres du groupe. De cette masse de souvenirs communs, et qui s'appuient l'un sur l'autre, ce ne sont pas les mêmes qui apparaîtront avec le plus d'intensité à chacun d'eux. Nous dirions volontiers que chaque mémoire individuelle est un point de vue sur la mémoire collective, que ce point de vue change suivant la place que j'y occupe, que cette place elle-même change suivant les relations que j'entretiens avec d'autres milieux. Il n'est donc pas étonnant que, de l'instrument commun, tous ne tirent pas le même parti. Cependant lorsqu'on essaie d'expliquer cette diversité, on en revient toujours à une combinaison d'influences qui, toutes, sont de nature sociale $»^{48}$.

Ce détour par l'espace relationnel dans lequel est pris l'individu permet ainsi à Halbwachs d'articuler l'individu et le collectif. Ce n'est pas le « groupe » en tant que groupe qui explique la mémoire collective mais la position de l'individu au sein de l'espace social comme l'évolution de ce dernier :

\footnotetext{
${ }^{45}$ HALBWACHS, 1997 [1950 posthume], p. 63.

${ }^{46}$ Sur ce point, voir le texte éclairant de Jean-Pierre Cléro dont est extrait cette phrase : « il existe des liens entre l'espace et le temps en ce sens que l'on peut « réaliser» par l'espace ce qu'on ne peut réaliser par le temps », Clero, 2008, p. 66.

${ }^{47}$ JAISSON, 1999.

${ }^{48}$ HALBWACHS, 1997 [1950 posthume], p. 94-95.
} 
«La succession de souvenirs, même de ceux qui sont les plus personnels, s'explique toujours par les changements qui se produisent dans nos rapports avec les divers milieux collectifs, c'est-à-dire, en définitive, par les transformations de ces milieux, chacun pris à part, et de leur ensemble $»^{49}$.

Ici, le renversement de perspective est total par rapport à la lecture dominante de leur «père fondateur $\gg$ qui caractérise les memory studies :

«Ce qu'on appelle le sentiment de l'unité de notre moi, où l'on voit quelques fois un principe original de cohésion des états n'est au fond que la conscience que nous prenons à chaque instant d'appartenir à la fois à divers milieux: mais elle n'existe que dans le présent. Comment subsisterait-elle pour des états rejetés dans le passé alors que la pression des milieux sociaux n'interviendrait plus ici ? $»^{50}$.

Etudier la mémoire collective, soit comprendre sa dynamique, s'est d'abord porter attention à la structure de l'espace social, tout à la fois matériel, symbolique et cognitif, dans lequel est pris celui qui se souvient comme à la manière dont celle-ci se modifie. Pour Halbwachs, s'intéresser à la mémoire ce n'est donc pas constituer un objet spécifique dont le traitement nécessiterait l'utilisation de méthodes et de concepts propres. C'est, au contraire, traiter, par d'autres voies, de la morphologie sociale, question fondamentale pour les sociologues de son temps, et préciser ainsi certaines des intuitions d'Emile Durkheim, y compris pour les amender $^{51}$. Ce lien entre morphologie et mémoire, entre société et esprit - traverse ainsi l'ensemble de son œuvre ${ }^{52}$. Il est explicité à plusieurs reprises par Halbwachs comme dans cet

\footnotetext{
${ }^{49}$ Idem, p. 95.

${ }^{50}$ Idem, p. 89.

51 JAISSON et MARCEL, 2005 ; BRIAN et JAISSON, 2005a. A plusieurs reprises, Halbwachs reviendra sur cette filiation durkheimienne, comme en conclusion du $3^{\text {ème }}$ chapitre des Cadres sociaux de la mémoire: «Durkheim a bien vu et bien distingué ces deux aspects de la société. S'il a insisté d'abord sur l'aspect contrainte, c'est qu'au début d'une science, il faut définir provisoirement les faits par des signes extérieurs faciles à saisir. Comme le sentiment de joie exprime, lorsqu'il résulte chez l'homme de l'action de la société, il y a coïncidence et fusion partielle entre les tendances individuelles et la coutume sociale, et le sentiment de peine ou de contrainte, au contraire, qu'il y a entre elles une opposition au moins partielle, il a dit qu'on reconnaîtrait les faits sociaux à ce qu'ils s'imposent à nous et nous contraignent. Mais il a reconnu qu'il n'y a pas de pratique collective qui n'exerce sur nous une double action, que les forces sociales s'orientent souvent dans le sens de nos désirs, qu'en tout cas elles accroissent et enrichissent notre être individuel de tous les modes de sensibilité et de toutes les formes de pensée que nous empruntons aux autres hommes », HALBWACHS, 1994 [1925], p. 112. L'importance de ce souhait de mener à sa pleine maturité l'œuvre durkheimienne explicite tout à fait différemment l'origine de l'intérêt d'Halbwachs pour la mémoire que la thèse du supposé refoulement du traumatisme de la Première Guerre mondiale défendue par Annette BECKER, 2003, sur ce point notamment, VERRET, 1972 ; BRIAN et JAISSON, 2005b ; TOPALOV, 2006b. Sur l'imbrication déjà centrale chez Durkheim entre morphologie et représentation, BELLAH, 1959.

${ }^{52}$ Sur cette question également, il existe un profond malentendu : « his writings display his thorough grounding in economics, not to mention a passion for satistical analysis seemingly at odds with the focus on imagery and imagination that the topic of memory requires », HUTTON, 1993, p. 74.
} 
extrait de La Morphologie sociale: «La morphologie sociale, comme la sociologie, porte avant tout sur des représentations collectives. Si nous fixons notre attention sur ces formes matérielles, c'est afin de découvrir derrière elles, toute une partie de la psychologie collective. Car la société s'insère dans le monde matériel, et la pensée du groupe trouve, dans les représentations qui lui viennent de ces conditions spatiales, un principe de régularité et de stabilité, tout comme la pensée individuelle a besoin de percevoir le corps et l'espace pour se maintenir en équilibre $»^{53}$.

Très (trop) rapidement esquissée $i^{54}{ }^{54}$, la voie ouverte par la théorie de la mémoire d'Halbwachs constitue donc une réponse presque terme à terme aux interrogations soulevées par le memory boom, elles-mêmes à l'origine de l'institutionnalisation des memory studies comme un champ de recherche intégré. La conception d'Halbwachs porte en elle l'approche interactionniste de la mémoire collective aujourd'hui tant attendue en ce qu'elle permettrait de dépasser une approche réifiante encore dominante. La conceptualisation de la mémoire collective proposée par Halbwachs permet également un dépassement de l'énigme de la « réception », identifiée comme la principale boîte noire des études contemporaines sur la mémoire $^{55}$. Dès lors que la mémoire est pensée comme le produit conscientisé de l'espace social dans lequel se situe l'individu, un paradigme relationnel se substitue logiquement à celui de la réception d'un récit du passé qui serait produit en dehors de cette relation ${ }^{56}$. Dans une perspective fondamentalement interactionniste, l'attention est alors portée au jeu processuel d'images réciproques qui préside à l'appropriation complexe du récit du passé.

Dans les années 60, Roger Bastide a peut-être été le premier à prendre conscience de la nature opératoire de la théorie sociologique d'Halbwachs pour travailler de manière empirique sur la mémoire $^{57}$. La perspective est alors totalement inversée par rapport à celle qui préside actuellement à l'institutionnalisation des memory studies. La mémoire collective est pensée comme un objet de sociologie générale dont la dynamique relève d'abord de processus

\footnotetext{
${ }^{53}$ HALBWACHS, 1946 [1938], p. 18.

${ }^{54}$ AMIOT, 1991 ; SABOURIN, 1997 ; LAVABRE, 1998.

${ }^{55}$ KANSTEINER, 2002 ; SChUMAN ET SCHWARTZ, 2005 ; Gudehus et al., $2010 \mathrm{~b}$.

${ }^{56}$ A ce stade, la nature problématique du terme récurrent de « contexte social », visiblement avancé sans aucune réflexion sur les structures, dans les études contemporaines de mémoire collective apparaît clairement.

${ }^{57}$ BASTIDE, 1970, 1995[1960] et 1996[1967]. En disant cela, nous avons conscience de contribuer à forger une généalogie alternative à celle des memory studies, LAVABRE, 2004. Il nous est ainsi impossible de nous affranchir ici des rapports de pouvoir et de concurrence, notamment disciplinaire, qui structure le champ. Au contraire, nous plaidons pour un retour réflexif de chacun sur sa propre position à l'égard de cet enjeu puisque ce retour est, à nos yeux, une des conditions de la production contemporaine d'un savoir cumulatif. Nous revenons sur ce point dans la dernière partie de cet article.
} 
morphologiques qu'il est possible de cerner à l'aide des méthodes et concepts ordinaires de sa discipline.

Quelques recherches récentes s'inscrivent également dans cette perspective et mobilisent la théorie halbwachsienne pour travailler sociologiquement sur la mémoire. Elles se distinguent cependant des travaux de R. Bastide sur un point fondamental. Alors que celui-ci écrivait dans les années 60 et 70, l'utilisation contemporaine des écrits d'Halbwachs débute, elle, dans les années 90, soit une fois le «memory boom» commencé. Ce faisant, elle prend acte de l'intérêt central et explicite des études de mémoire collective - qui se multiplient alors depuis déjà plusieurs années - pour la question des « commémorations », des « usages du passé » et autres « politiques de la mémoire » qui, on l'aura compris, n'est pas en tant que telle ce qui intéresse Halbwachs, en ce que celui-ci se place d'abord à l'échelle de l'individu social ${ }^{58}$.

Se saisissant de manière opératoire de la proposition halbwachsienne, plusieurs chercheurs ont ainsi construit des objets de recherche au croisement des mémoires exprimées par les individus et des tentatives institutionnelles et politiques les encadrer ${ }^{59}$. Pionnière de cette mobilisation contemporaine des travaux d'Halbwachs, Marie-Claire Lavabre a clairement résumé cette perspective « résolument sociologique » :

Celle-ci «tire la mémoire, non du côté des souvenirs individuels entendus comme témoignages et récits des événements vécus ou des usages du passé tels que les commémorations et les monuments les donnent ponctuellement à voir, mais du côté des mécanismes de la socialisation, familiale, scolaire ou partisane, du côté de la logique interne des représentations du passé telle que l'exprime la durée de la mémoire historique d'un groupe et telle que la restituent les individus $»{ }^{60}$.

En d'autres termes, et dans une perspective renversée par rapport à celle que défendent les travaux de J. Winter et E. Sivan, il s'agit de cerner et de comprendre « l'homo sociologicus » qui se cache derrière tout «homo actans ». Cette étude halbwachsienne contemporaine de l’homo sociologicus qui préside à la dynamique de la mémoire collective a été menée à

\footnotetext{
${ }^{58}$ Sur ce point aussi, un important malentendu persiste sur l'œuvre halbwachsienne. « Halbwachs showed historians how to write history of the politics of memory, and it is especially for this accomplishment that they pay him homage today », HUTTON 1993, p. 75. Cette lecture fréquente explique que l'échelle des commémorations publiques et donc la constitution de corpus faits majoritairement de sources officielles, imprimées et immédiatement disponibles, soient considérées comme avoir été privilégiées par le sociologue français. Sans que la question de savoir si ces «commémorations » sont la manifestation ou le vecteur de la mémoire collective, LAVABRE, 1991 ; ROUSSO, 1991, celles-ci sont censées manifester, d'une manière ou d'une autre, l'existence de cette « mémoire », pensée donc comme transcendante et a priori consensuelle.

${ }^{59}$ LAVABRE, 2001 ; GENSBURGER, 2008a.

${ }^{60}$ LAVABRE, 1994a, p. 168.
} 
travers des objets et à des échelles diverses ${ }^{61}$. Ces chercheurs qui se réclament de la théorie sociologique d'Halbwachs portent à chaque fois, et d'abord, attention aux structures des relations sociales auxquelles participent les individus qui se « souviennent ».

Ce faisant, ces recherches ont démontré, à chaque fois, que les politiques commémoratives et autres actions institutionnelles relatives à la mémoire ne produisent pas d' « effets » linéaires mais sont l'objet d'appropriations sociales complexes. La mémoire portée et exprimée par les individus ne découlent pas tant d'un rejet ou d'une acceptation d'un récit du passé prescrit que de l'espace social relationnel au sein desquelles ils se situent comme de son évolution. C'est avant tout et seulement parce que l'individu se reconnaît possiblement dans le point de repère offert par les institutions que le récit du passé proposé par ces dernières « influence » sa mémoire et ce toujours de manière polysémique et non-linéaire. Prolongements contemporains de la théorie d'Halbwachs, ces quelques travaux conduisent ainsi à renverser radicalement les questionnements qui président à la disciplinarisation actuelle des memory studies. Outre qu'ils ouvrent la voie à un dépassement de la réification de la mémoire collective, ils invitent à substituer l'étude des processus d'appropriations et de jeux d'images réciproques à celle des phénomènes de «réception » et leurs corollaires que sont ceux de «production ».

En sus de ses conséquences théoriques, un tel retournement de perspective a, bien entendu, des implications méthodologiques fondamentales ${ }^{62}$. La première concerne l'échelle à laquelle conduire l'enquête. S'inspirer de la grille d'analyse halbwachsienne suppose en effet de s'intéresser à la manière dont les individus construisent leur rapport au passé, et réciproquement, au fait que les institutions de mémoire et autre commémoration sont ellesmêmes faites par des individus. L'adoption d'une telle focale a ensuite une conséquence méthodologique fondamentale. Loin de l'étude de la «mémoire» par son écume ${ }^{63}$, elle nécessite la conduite d'enquêtes ethnographiques approfondies, mêlant entretiens

\footnotetext{
${ }^{61}$ Par exemple Lavabre, 1994a ; Gensburger, 2005 et 2010 ; Oeser, 2010 ; Garcia Castro, 2002 ; LABORDE, 2005.

62 Sur ce point, les intuitions d'Halbwachs ne sont aujourd'hui réellement opératoires que si elles s'accompagnent de la mise en œuvre de protocoles d'enquête étrangers, eux, à l'auteur de la Mémoire collective comme d'ailleurs à la plupart des sociologues de son temps. Si Halbwachs emprunte de nombreux exemples à sa vie personnelle, à la littérature ou à l'imagination, il déclare lui-même avoir «l'esprit un peu trop tourné aux abstractions », lettre du 27 février 1927 à Marcel Mauss citée par Jean-Christophe MARCEL, 2004, p. 16. Sur le rapport d'Halbwachs à l'empirie voir également TOPALOV, 1999 et 2006a.

${ }_{63} \mathrm{Au}$ fur et à mesure du développement du «memory boom», les traces matérielles de la commémoration en sont ainsi peu à peu venues à incarner la mémoire et non plus en être le supposé «vecteur », KLEIN, 2000. Robert Wuthnow justifie lui une telle approche en prétendant qu'on ne peut jamais savoir ce que ces supports matériels signifient pour les individus, WUTHNOW, 1987. Nous défendons au contraire l'idée que construire des objets qui permettent cette compréhension constitue précisément la tâche, certes difficile et toujours inaboutie, du sociologue.
} 
compréhensifs et terrains d'observation, y compris participante, et leur croisement avec le dépouillement d'archives et l'étude, elle largement répandue, de textes et de publications officiels. Les travaux qui viennent d'être cités mobilisent ainsi systématiquement des corpus ethnographiques diversifiés. Pour cela, les outils méthodologiques ordinaires de la discipline s'avèrent ainsi tout à fait pertinents tant est que l'on est prêt à les appliquer à l'étude de la mémoire collective.

\section{Des cadres sociaux de la mémoire à la mémoire comme cadre social}

Il est donc possible de s'appuyer effectivement, et de manière empiriquement opératoire, sur l'œuvre de M. Halbwachs pour défendre une autre modalité de renouvellement des travaux sur la mémoire. Faire un tel choix implique de doublement banaliser le traitement de l'objet «mémoire »: en s'affranchissant des nombreuses considérations normatives qui le soustendent aujourd'hui, d'une part, et en mobilisant les outils ordinaires des sciences sociales, de l'autre $^{64}$. En rupture avec l'étude quasi-exclusive des commémorations et autres «lieux de mémoire », cela passe par la prise en compte de l'individu social comme lieu de la mémoire. Cette approche suppose une réflexion systématique sur la construction d'objets intermédiaires, les sources mobilisées, les méthodes employées et les échelles adoptées.

Suivre cette voie alternative aux memory studies compte, cependant, un préalable. Il convient en effet de comprendre l'usage que les protagonistes de cette disciplinarisation font d'Halbwachs et, plus largement, de cerner les enjeux qui sous-tendent le phénomène en cours. Sans avoir la prétention de réaliser ici une véritable archéologie des memory studies ${ }^{65}$, nous souhaitons ouvrir quelques pistes en ce sens et plaider pour davantage de réflexivité, en premier lieu disciplinaire, de la part des chercheurs qui travaillent sur la mémoire collective, réflexivité indispensable à la construction cumulative d'un savoir le plus souvent présenté comme « inter » voir « trans » disciplinaire.

\footnotetext{
${ }^{64}$ Gensburger et LAVABRE, 2005.

${ }^{65}$ L'étude de la genèse des « urban studies » ou « études urbaines » montre pourtant la pertinence qu'il y aurait à entreprendre une telle recherche, RHSS, 2005 ; VAN DAMME, 2005 ; TOPALOV, 2008. De même la publication récente d'une étude généalogique de la revue Sociologie du travail (Paris) indique l'intérêt qu'il y aurait à prendre comme point d'entrée l'étude de la revue Memory Studies dans une telle perspective, BORZEIX et ROT, 2010 .
} 
De toute évidence, la référence à Halbwachs comme au père fondateur des memory studies joue tout d'abord un rôle stratégique pour la réussite de cette nouvelle étiquette disciplinaire ${ }^{66}$. Elle permet d'attester d'une généalogie. Un mécanisme semblable a déjà été utilisé au cours des années 80 pour légitimer le fait de s'intéresser à la mémoire, par exemple pour la sociologie américaine contemporaine ${ }^{67}$. Cet usage stratégique d'un Halbwachs - Père Fondateur n'explique pas cependant la lecture spécifique, et largement décalée, qui en est faite par les artisans de la disciplinarisation en cours. Comment comprendre que la reprise de l'œuvre de l'auteur de la Mémoire collective se fasse sur un mode tellement éloigné du contenu de celle-ci?

Une première raison peut être envisagée. Principalement le fait de chercheurs anglo-saxons, cette mention d'Halbwachs pour légitimer les memory studies a lieu alors même que ses ouvrages sur la mémoire n'ont été que très partiellement traduits et que le reste de ses écrits sur la ville, les classes sociales et la morphologie - dont nous avons vu le lien étroit avec les précédents -1 'a été encore moins ${ }^{68}$. Mais cette explication est très insuffisante. Une simple recherche dans la base de données de JSTOR fait tout d'abord apparaître que, à qui chercherait à lire effectivement Halbwachs dans le texte, celui-ci a publié de son vivant et en anglais plusieurs textes dont deux articles de psychologie collective dans chacune des grandes revues américaines de sociologie ${ }^{69}$ tandis que ses ouvrages sur la mémoire ont fait l'objet de comptes-rendus dès leurs publications. En 1925, l'auteur, enthousiaste, de celui des Cadres sociaux de la mémoire conclut, par exemple, son propos en un sens radicalement différent de celui qui fait d'Halbwachs l'inventeur d'un champ de recherche thématique et interdisciplinaire: «Students of general sociological theory and methodology will be interested in this study $»^{70}$.

Ensuite, la nature formelle et décalée de la référence actuelle à Halbwachs par les tenants de l'institutionnalisation des memory studies se retrouve à l'identique chez la plupart des

\footnotetext{
${ }^{66}$ TOPALOV, 2004.

${ }^{67}$ SCHWARTZ, 1982 et 1996.

${ }^{68}$ Lewis Coser a traduit en 1992 des extraits de La Topographie Légendaire des Evangiles en Terre Sainte. Etude de mémoire collective et des Cadres Sociaux de la Mémoire, HALBWACHS, 1992. L'édition posthume de la Mémoire collective a elle été traduite en 1980 et publiée avec une introduction de Mary Douglas, HALBWACHS, 1980. Le reste des travaux de Maurice Halbwachs a été très peu traduit, HALBWACHS, 1958, 1960 et 1978. En Allemagne, si la traduction est elle presque systématique, elle s'effectue sur un mode binaire qui distingue nettement les travaux sur la mémoire du reste de son œuvre, EGGER et SCHULTHEIS (eds.), 2001 à 2003 d'un côté ; GUDHEUS et al., 2010 de l'autre, du fait notamment de la distinction traditionnelle entre matérialisme et idéalisme, JAISSON et MARCEL, 2005, p. 101.

${ }^{69}$ HALBWACHS, 1938 et 1939.

${ }^{70}$ House, 1925, p. 392.
} 
historiens français qui, depuis les années 80, étudient la mémoire collective. Si nombreux sont ceux qui citent l'ancienneté des travaux d'Halbwachs, là encore aucun ne mobilise ses propositions conceptuelles dans le cœur de leurs travaux. L'évolution de la mention d'Halbwachs par Pierre Nora est à cet égard exemplaire. Dans un de ses tout premiers textes sur ce qui deviendra «l'histoire de la mémoire », il reconnaît la paternité d'Halbwachs sur le terme de 《mémoire collective $»^{71}$. Mais en note, il renvoie à une citation erronée de l'œuvre. Le titre de l'ouvrage posthume de 1950 y est porté au pluriel (Les Mémoires Collectives). Dans l'un des tomes des Lieux de mémoire, il fera à nouveau référence à Halbwachs pour préciser que « la redécouverte, au cours des années soixante-dix, de l'œuvre déjà ancienne, Les Cadres sociaux de la mémoire, ou sa réédition, La Mémoire collective, rééd. 1968, sont précisément l'effet, plutôt que la cause, de l'intérêt nouveau des historiens pour la mémoire $»^{72}$. Là encore la superficialité de la lecture du texte halbwachsien saute aux yeux. La Mémoire collective n'est en rien une réédition des Cadres sociaux, bien au contraire ${ }^{73}$.

Or il peut être fait l'hypothèse que c'est d'abord, et principalement, à travers l'œuvre de P. Nora - elle rapidement, et largement, traduite et diffusée dans le monde anglo-saxon - qu'est «lue» celle d'Halbwachs. Plusieurs des auteurs fondateurs de l'étude de la mémoire collective aux Etats-Unis comme aujourd'hui les défenseurs de l'institutionnalisation des memory studies rapprochent ainsi systématiquement les deux auteurs. En 1993 déjà, 1'historien P. Hutton établit cette équivalence dans le chapitre qu'il consacre à Halbwachs :

« The most direct and imposing contemporary application of Halbwachs's method, however, is the study of the representation of the French national memory, edited by Pierre Nora, Les Lieux de mémoire. Acknowledging the significance of Halbwachs's work directly, Nora follows his method in working backward from the present - upstream if you will - to inventory the many traditions that have enshrined the French national memory $\gg{ }^{74}$.

Cinq ans plus tard, dans l'un des premiers bilans des études de mémoire collective, les sociologues J.K. Olick et J. Robbins procèdent à un rapprochement identique :

«French historian Pierrre Nora (1992), leading theoretician and editor of a massive sevenvolume project on «places » or « lieux » of French memory, also, begins by observing the paradoxes of memory in postmodernity. 'We speak so much of memory', he writes, 'because there is so little of it left'. Nora can in this way be seen as the true heir to Halbwachs who

\footnotetext{
${ }^{71}$ NORA, 1978.

${ }^{72}$ NORA, 1997, p. 4718.

${ }^{73}$ SABOURIN, 1997 ; JAISSON, 1999. P. Nora est revenu depuis sur son rapport à l'œuvre d'Halbwachs, NoRA, 2003.

${ }^{74}$ Hutton, 1993, p. 88.
} 
noted the passing of memory into history as we lose a living relation to the past, though Nora sees this process as even more dramatic and irreversible, and as more clearly politicial, than Halbwachs did $»^{75}$.

Plus récemment, dans les débats qui ont accompagné la naissance des memory studies, ce rapprochement perdure. Il est question ici de «Pierre Nora's work in the tradition of Halbwachs $»^{76}$. Il est fait là allusion à la nature complémentaire de leurs travaux :

«Drawing on sources including, but by no means limited to Halbwachs's seminal sociological investigations of the role of social institutions in the transmission of memory (1980) and Pierre Nora's multi-volume study of French memory places (Nora 1984, 19961998), memory research has coined a wealth of concepts to describe memory that exceeds the personal $»^{77}$.

L'appropriation d'Halbwachs par les chercheurs qui se regroupent aujourd'hui pour constituer un champ des memory studies est donc susceptible de s'être effectuée au second degré, à partir d'une lecture systématique des « historiens de la mémoire », au premier rang desquels P. Nora. Les usages d'Halbwachs manifesteraient alors les rapports entre disciplines qui se jouent à travers ce champ de recherche interdisciplinaire ${ }^{78}$. Plus précisément, ils résulteraient largement de la domination historienne en la matière comme, dans l'exact même temps, de la tentative des autres disciplines de s'en émanciper, au moins partiellement. Il est ainsi intéressant de constater que c'est un sociologue, J.K. Olick, qui se retrouve le plus souvent au côté du label de «memory studies » dans les publications indexées dans JSTOR depuis 2007 (voir Annexe). L'entreprise de disciplinarisation que constitue l'émergence des memory studies doit ainsi être étudiée à la lumière des conflits de légitimité entre disciplines mais

\footnotetext{
${ }^{75}$ OLICK et RoBbins, 1998, p. 120-121.

${ }^{76}$ KANSTEINER, 2002, p. 183.

77 RADSTONE, 2008, p. 33. Cette lecture d'Halbwachs à travers l'œuvre de P. Nora n'est pas propre aux chercheurs anglo-saxons. Il est ainsi frappant de constater qu'alors que le sociologue Michael Pollak réalise une enquête qui rencontre pourtant, et sur plusieurs points, la théorie halbwachsienne, POLLAK, 1990 ; GENSBURGER, 2008b, lorsqu'il cite explicitement Halbwachs c'est finalement pour l'identifier à P. Nora : « dans son analyse de la mémoire collective, Maurice Halbwachs met l'accent sur la force des différents repères qui structurent notre mémoire et qui l'insèrent dans celle de la collectivité à laquelle nous appartenons. En font partie bien évidemment les monuments, ces lieux de mémoire analysés par Pierre Nora, le patrimoine architectural et son style qui nous accompagnent tout au long de notre vie, les paysages, les dates et personnages historiques dont on nous répète inlassablement l'importance [...] Dans la tradition européenne du XIX siècle, y compris chez Halbwachs, la nation est la forme la plus achevée d'un groupe, et la mémoire nationale la forme la plus accomplie d'une mémoire collective », POLLAK, 1993, p. 14-15. Pour une analyse spécifique de ce décalage, LAVABRE, 2008.

${ }^{78}$ LAVABRE, 1994 b.
} 
aussi probablement entre traditions nationales, lutte à laquelle nous sommes d'ailleurs consciente de participer - fût-ce en réfutant la pertinence même du champ ${ }^{79}$.

Ainsi lorsque, dans un des articles plébiscites des premiers numéros de Memory Studies, H.L. Roediger et J.V. Wertsch plaident pour la création d'une «new discipline », ils dressent simultanément la liste des disciplines existantes intéressées à ce débat à titre principal. L'histoire, la littérature, la philosophie, la psychologie et les sciences de l'éducation se voient ainsi saluées comme «the core disciplines for a new field of memory studies ${ }^{80}$. Certes évoquées, et dans cet ordre, la science politique, l'architecture, le droit, la sociologie/media studies/communication, l'économie, les neurosciences et l'anthropologie se voient attribuées, elles, un statut secondaire. En 2002, dans un article critique du «memory boom», W. Kansteiner, spécialiste des media and cultural studies, appelait déjà à la création d'un champ interdisciplinaire. Il insistait simultanément sur le fait que les «historians of collective memory can profit from the sophisticated discussions about reception and audience behavior in media and cultural studies $\gg{ }^{81}$.

Là encore, le renversement est total par rapport à la démarche d'Halbwachs. Pourtant authentiquement compétent dans de nombreuses autres disciplines que la sienne ${ }^{82}$, Halbwachs s'est intéressé à la mémoire d'un point de vue sociologique comme à un objet pertinent pour fonder la pertinence de sa discipline notamment au regard de l'histoire ${ }^{83}$ et de la psychologie ${ }^{84}$. Aujourd'hui des chercheurs issus de disciplines différentes revendiquent une pratique interdisciplinaire pour en définitive affirmer la primauté de leur discipline pour travailler sur un objet «mémoire » jugé, lui, spécifique. Alors que dans le premier cas, la perspective est celle de la sociologie générale dont la mémoire n'est qu'une porte d'entrée thématique pour toucher du doigt le social, ici c'est l'expertise disciplinaire sur la «mémoire » en tant que telle qui est l'objet d'une concurrence disciplinaire sous couvert d'interdisciplinarité.

\footnotetext{
${ }^{79}$ Nous parlons en effet depuis une sociologie qui combine travail de terrain et réflexion épistémologique, d'une part, un univers d'abord francophone, de l'autre.

${ }^{80}$ RoEDiger et Wertsch, 2008, p. 14.

${ }^{81}$ KANSTEINER, 2002, p. 195.

${ }^{82}$ Des mathématiques, BRIAN (Ed.), 2010, à l'histoire, BRIAN et JAISSON, 2005a, en passant par la philosophie dans laquelle, comme tous les sociologues de son époque, il avait été formé à l'origine, HALBWACHS, 1906 ; CLERO, 2008.

${ }^{83}$ BLOCH, 1925. Comme le fait remarquer J-P. Cléro « ce n’est pas tant la distinction entre histoire et mémoire en tant que matière qu'Halbwachs aborde que celle entre histoire et sociologie en tant que disciplines », CLERO, 2008, p. 59.

${ }^{84}$ MuCChIELli, 1999 ; HalbWaCHS, 1929.
} 
Ces quelques éléments conclusifs invitent donc à élaborer une véritable sociologie des memory studies qui porterait attention à la position des acteurs au sein de l'espace social qu'est le paysage académique. Ils conduisent également à s'interroger sur les raisons pour lesquelles ce champ se développe aujourd'hui et donc à comprendre comment la « mémoire » est devenue une ressource et un enjeu de connaissance mais aussi d'expertise dans une société où le statut de la recherche scientifique connaît, depuis quelques années, une profonde mutation. A l'opposé des présupposés qui président aujourd'hui à l'institutionnalisation des memory studies, l'héritage halbwachsien invite ainsi à porter attention non seulement aux cadres sociaux de la mémoire mais aussi à la manière dont la "mémoire » est effectivement devenue un cadre social, y compris pour la production du savoir.

\section{Références}

Амiот (Michel), 1991, « Le Système de pensée de Maurice Halbwachs », Revue de Synthèse, 2, p. $265-288$.

Armstrong (Elizabeth A.) et Suzanna Crage, 2006, « Movements and Memory: The Making of the Stonewall Myth », American Sociological Review, 71, p. 724-751.

BASTIDE (Roger), 1995 [1960], Les Religions africaines au Brésil, Paris, PUF.

BAstide (Roger), 1996 [1967], Les Amériques Noires, Paris, L’Harmattan.

BASTIDE (Roger), 1970, «Mémoire collective et sociologie du bricolage », L'Année sociologique, p. 65-108.

BAudelot (Christian) et Roger Establet, 1994, Maurice Halbwachs. Consommation et société, Paris, PUF.

BECKER (Annette), 2003, Maurice Halbwachs : un intellectuel en guerres mondiales 19141945, Paris, Viénot.

Bellah (Robert N.), 1959, «Durkheim and History », American sociological review, 24(4), p. $447-461$.

BERLINER (David), 2005, «The abuses of Memory : reflections on the Memory Boom in Anthropology », Anthropological Quarterly, 78(1), p. 197-211.

Bloch (Marc), 1925, «Mémoire collective, tradition et coutume. A propos d'un livre récent », Revue de synthèse historique, XIV (118-120), p. 73-83.

BorzeIX (Annie) et Gwenaële Rot, 2010, Genèse d'une discipline, naissance d'une revue : Sociologie du travail, Nanterre, Presses Universitaires de Paris Ouest. 
BriAn, (Eric) (Ed.), 2010, « Introduction », HALBWACHS (Maurice), LA THEORIE DE L'HOMME MOYen. Essai Sur QueTelet eT LA STATISTIQUe MORALE, Paris, Sciences en situations.

BRIAN (Eric), 2008, « Portée du lexique halbwachsien de la mémoire ». In M. Halbwachs, La Topographie Légendaire des Evangiles en Terre Sainte, Paris, PUF, réédition dirigée par Marie Jaisson, p. 113-145.

BRIAN (Eric) et Marie JAISSON, 2005a, «Nombre et mémoire. Halbwachs sociologue probabiliste », dans KRAPOTH (Hermann) et Denis LABORDE (Ed.), Erinnerung und Gesellschaft. Mémoire et société, Wiesbaden, VS Verlag fur Sozialwissenschaften, p. 127151.

BRIAN (Eric) et Marie JAISSON, 2005b, « Probability, biology and sociology in the human sexratio at birth. A note on the trace of the First World War»,Journ@l Electronique d'Histoire des Probabilités et de la Statistique, 1, 1.

BrockmeIER (Jens), 2010, “Cultural Memory Studies : An International and Interdisciplinary Handbook. A review", Memory Studies, 3, p. 74-76.

Brown (Adam D.) et al., 2009, «Is an Interdisciplinary Field of Memory Studies Possible ? », International Journal of Politics, Culture and Society, 22, p. 117-124.

Clero (Jean-Pierre), 2008, « Halbwachs et l'espace fictionnel de la ville », dans HALBWACHS (Maurice), La Topographie Légendaire des Evangiles en Terre Sainte, Paris, PUF, réédition dirigée par Marie Jaisson, p. 43-72.

Confino (Alon), 1997, "Collective Memory and Cultural History: Problems of Method", American Historical Review, 105, p. 1386-1403.

Coser (Lewis. A.), 1992, "The Revival of the Sociology of Culture : The Case of Collective Memory", Sociological Forum, 7(2), p. 365-373.

CRANE (Susan), 1997, «Writing the Individual Back into Collective Memory », American Historical Review, 102(5), p. 1372-1385.

Deloye (Yves) et Claudine Haroche (Eds.), 2004, Maurice Halbwachs. Espaces, mémoires et psychologie collective, Paris, Publications de la Sorbonne.

EGGER (Stephan) et F. SCHUlTHEIS (Eds.), 2001a, Entwurf einer Psychologie sozialer Klassen über die gesellschaftlichen Antriebe des Menschen, UVK - Université de Konstanz, tome 1.

EGGer (Stephan) et Franz SchultheIs (Eds.), 2001b, Klassen und Lebensweisen, UVK Université de Konstanz, tome 2.

EGGer (Stephan) et Franz SchUltheIS (Eds.), 2001c, Kollektive Psychologie, UVK Université de Konstanz, tome 3.

EgGer (Stephan) et Franz Schultheis (Eds.), 2002, Soziale Morphologie, UVK - 
Université de Konstanz, tome 4.

EgGer (Stephan) et Franz SchultheIs (Eds.), 2001d, Theorie und Methode, UVK Université de Konstanz, tome 5.

EGGER (Stephan) et Franz SchultheIS (Eds.), 2003, Stätten der Verkündigung im Heiligen

Land. Eine Studie zum kollektiven Gedächtnis, UVK - Université de Konstanz, tome 6.

EGGER (Stephan), 2003, Maurice Halbwachs. Aspekte des Werks, UVK - Université de Konstanz.

Erll (Astrid) et Ansgar Nunning (Eds.), 2008, A Companion to Cultural Memory Studies. An International and Interdisciplinary Handbook, Berlin/New York, Walter de Gruyter.

FALASCA ZAMPONI (Simonetta), 1998, «Of Storytellers and Master Narratives. Modernity, Memory and History in Fascist Italy », Social Science History, 22(4), p. 415-444.

FIVUSH (Robyn), 2010, «Cultural Memory Studies : An International and Interdisciplinary Handbook. A review", Memory Studies, 3, p. 76-78.

Garcia Castro (Antonia), 2002, La mort lente des disparus au Chili. Sous la négociation civils-militaires (1973-2002), Paris, Maisonneuve \& Larose.

Gedi (Noa) et Yigal Elam, 1996, “Collective Memory - What Is It ?”, History and Memory, $8(1)$, p. $30-50$.

Gensburger (Sarah), 2010, "From the memory of rescue to the institution of the title of Righteous among the Nations », dans ANDrIEU (Claire), Sarah Gensburger et Jacques SEmelin (Eds.), Resisting Genocides, London/New York, Hurst/Columbia University Press, p. 19-32.

Gensburger (Sarah), 2008b, « Utiliser la sociologie de Michael Pollak aujourd'hui ? Retour sur deux études de cas », dans IsRAËL (Liora) et Danièle Voldman (Eds.), Michael Pollak. De l'identité blessée à une sociologie des possibles, Bruxelles/Paris, Complexes/CNRS Editions, p. 127-141.

Gensburger (Sarah), 2008a, «Fragments de mémoire collective : les Justes parmi les Nations ", HALBWACHS (Maurice), La Topographie légendaire des évangiles en terre sainte. Etude de mémoire collective, Paris, PUF, réédition dirigée par Marie Jaisson, p. 99-122.

Gensburger (Sarah) et Marie-Claire Lavabre, 2005, «Entre 'devoir de mémoire’ et 'abus de mémoire' : la sociologie de la mémoire comme tierce position », dans Muller Bertrand (Ed.), Histoire, mémoire et épistémologie. A propos de Paul Ricoeur, Lausanne, Payot, p. 76105.

Gensburger (Sarah), 2005, «Essai de sociologie de la mémoire : le cas des camps annexes de Drancy dans Paris », Genèses, 61, p. 47-69. 
Goody (Jack), 1977, « Mémoire et apprentissage dans les sociétés avec et sans écriture : la transmission du Bagre », L'Homme, 1, p. 29-52.

Gudehus (Christian), Stewart ANDERSON et David Keller, 2010b, « Understanding Hotel Rwanda : A reception study », Memory studies, 3(4), p. 344-363.

Gudehus (Christian), Ariane Eichenberg ET Harald Welzer (Eds.), 2010a, Gedächtnis und Erinnerung. Ein interdisziplinäres Handbuch, Stuttgart, J.B. Metzler.

Halbwachs (Maurice), 2008 [1941], La Topographie légendaire des évangiles en terre sainte. Etude de mémoire collective, Paris, PUF, réédition dirigée par Marie Jaisson.

Halbwachs (Maurice), 1997 [1950 Posthume], La mémoire collective, Paris, Albin Michel.

Halbwachs (Maurice), 1994 [1925], Les cadres sociaux de la mémoire, Paris, Albin Michel.

Halbwachs (Maurice), 1992, On Collective Memory, Chicago : University of Chicago Press.

Halbwachs (Maurice), 1980, The Collective Memory, New York, Harper and Row.

HaLBWACHS (Maurice), 1978, The causes of suicide, London, Routledge.

Halbwachs (Maurice), 1960, Population and society. Introduction to social morphology, Glencoe, Ill., Free Press.

HaLBWACHS (Maurice), 1958, The psychology of social class, London, Heinemann.

Halbwachs (Maurice), 1946 [1938], Morphologie sociale, Paris, Armand Colin.

HALBWACHS (Maurice), 1939, «Individual consciousness and collective mind », The American Journal of Sociology, 44(6), p. 812-822.

HALBWACHS (Maurice), 1938, «Individual psychology and collective psychology », American Sociological Review, 3(5), p. 615-623.

HALBWACHS (Maurice), 1929, « La psychologie collective d'après Charles Blondel », Revue critique, 107, p. 444-456.

HalBWACHS (Maurice), 1906, Leibniz, Paris, Delaplane.

House (Floyd N.), 1925, «Les Cadres Sociaux de la Mémoire », The American Journal of Sociology, 31(3), p. 390-392.

HutTon (Patrick. H.), 2010, «Cultural Memory Studies : An International and Interdisciplinary Handbook. A review”, Memory Studies, 3, p. 78-83.

HutTon (Patrick. H.), 1993, History as an Art of Memory, Hanover and London, University Press of New England.

JAISSON (Marie) et Christian BAudelot (Eds.), 2007, Maurice Halbwachs. Sociologue retrouvé, Paris, Editions de l'ENS. 
JAISSON (Marie) et Jean-Christophe MARCEL, 2005, « Morphologie et mémoire », Maurice Halbwachs (Maurice) et Alfred Sauvy, Le Point de vue du nombre, 1936, Paris, INED, p. 101-110, édition critique par Eric Brian et Marie Jaisson.

JAISSON (Marie), 1999, « Temps et espace chez Maurice Halbwachs. (1925-1945)», Revue d'histoire des sciences humaines, 1, p. 163-178.

JAISSON (Marie), 2008, « Mémoire collective et espace social ». HALBWACHS (Maurice), La Topographie légendaire des évangiles en terre sainte. Etude de mémoire collective, Paris, PUF, réédition dirigée par Marie Jaisson, p. 73-97.

JANSEN, (Robert S.), 2007, «Resurrection and Appropriation : Reputational Trajectories, Memory Work and the Political Use of Historical Figures », American Journal of Sociology, 112(4), p. 953-1007.

KAnsteIner (Wulf), 2002, «Finding Meaning in Memory : A Methodological Critique of Collective Memory Studies », History and Theory, 41(2), p. 179-197.

KLEIN (Kervin Lee), 2000, " On The Emergence of "Memory" in Historical Discourse », Representations, 69, p. 127-150.

LABORDE (Denis), 2005, La Mémoire et l'Instant. Les improvisations chantées du bertsulari basque, Bayonne, Elkar.

LAVABre (Marie-Claire), 2008, « Michaël Pollak et la mémoire collective ». dans ISRAËL (Liora) et Danièle Voldman (Eds.), Michael Pollak. De l'identité blessée à une sociologie des possibles, Bruxelles/Paris, Complexes/CNRS Editions, p. 115-126.

LAVABre (Marie-Claire), 2004, « Roger Bastide, lecteur de Maurice Halbwachs » Deloye (Yves) et Claudine Haroche (Eds.), Maurice Halbwachs. Espaces, mémoires et psychologie collective, Paris, Publications de la Sorbonne, p. 161-171.

LAVABRE (Marie-Claire), 2001, « De la notion de mémoire à la production des mémoires collectives » dans CEFAI Daniel (Ed.), Cultures politiques, Paris, Presses de la FNSP, p. 233252.

LAVABre (Marie-Claire), 1998, «Maurice Halbwachs et la sociologie de la mémoire », Raison Présente, 128, p. 47-56.

LAVABRE (Marie-Claire), 1994b, « Usages du passé, usages de la mémoire », Revue française de science politique, p. 480-493.

LAVAbre (Marie-Claire), 1994a, Le fil rouge. Sociologie de la mémoire communiste, Paris, Presses de la Fondation Nationale des Sciences Politiques.

LAVABRE (Marie-Claire), 1991, «Du poids et du choix du passé. Lecture critique du Syndrome de Vichy », Cahiers de l'IHTP, 18, p. 264-278. 
LEVy (Daniel), Jeffrey K. OLICK et al. (eds.), 2011, The Collective Memory Reader, Oxford, Oxford University Press.

MARCEL (Jean.-Christophe), 2004, «Mauss et Halbwachs: vers la fondation d'une psychologie collective (1920-1945) », Sociologie et sociétés, 36 (2), p. 73-90.

Martinez Gutierrez (Emilio) (Ed.), 2008, Maurice Halbwachs : Estudios de morfologia social de la ciudad, Madrid, CIS.

MONTLIBERT (Christian) (Ed.), 1997, Maurice Halbwachs. 1877-1945, Strasbourg, PUS.

MuCCHIELLi (Laurent), 1999, «Pour une psychologie collective: l'héritage durkheimien d'Halbwachs et sa rivalité avec Blondel durant l'entre-deux-guerres », Revue d'histoire des sciences humaines, 1, p. 103-141.

NAMER (Gérard), 2000, Halbwachs et la mémoire sociale, Paris, L'Harmattan.

NORA (Pierre), 2003, préface à Annette Becker, Maurice Halbwachs : un intellectuel en guerres mondiales 1914-1945, Paris, Viénot.

NoRA (Pierre), 1997 [1992], «L’ère de la commémoration» dans Les Lieux de mémoire, Paris, Gallimard.

Nora (Pierre), 1978, Mémoire collective, dans Le Goff (Jacques), Roger CharTIER et al. (Eds), La Nouvelle Histoire, Paris, CEPL.

OESER (Alexandra), 2010, Enseigner Hitler: les adolescents allemands face au passé nazi. Appropriations, Interprétations et usages de l'histoire, Paris, Editions de la MSH.

OLICK (Jeffrey.K), 2009, «Between Chaos and Diversity: Is Social Memory Studies a Field ? », International Journal of Politics, Culture and Society, 22, p. 249-252.

Olick (Jeffrey.K), 2008a, The Politics of Regret. On Collective Memory and Historical Responsability, New York, Routledge.

OLICK (Jeffrey.K), 2008b, « Collective memory : A memoir and prospect», Memory studies, 1, p. 23-29.

OLICK (Jeffrey.K), 2007, « Collective Memory and Nonpublic Opinion : A Historical Note on a Methodological Controversy About a Political Problem », Symbolic Interaction, 30 (1), p. $41-55$.

OLICK (Jeffrey.K), 1999b, “Collective Memory : the Two cultures”, Sociological Theory, 17 (3), p. 333-348.

OLICK (Jeffrey.K), 1999a, "Genre Memories and Memory Genre : A Dialogical analysis of May 8, 1945 Commemorations in the Federal Republic of Germany”, American Sociological Review, 64(3), p. 381-402. 
Olick (Jeffrey.K) et Joyce RobBInS, 1998, "Social Memory Studies : From « Collective Memory » to the Historical Sociology of Mnemonic Practices", Annual Review of Sociology, p. $105-140$.

PÉQuignot (Bruno) (Ed.), 2007, Maurice Halbwachs : le temps, la mémoire et l'émotion, Paris, L'Harmattan.

PollaK (Michael), 1993, «Mémoire, oubli, silence», dans PollaK (Michael) (Ed.), Une identité blessée. Etudes de sociologie et d'histoire, Paris, Métailié, p. 15-39.

PollaK (Michael), 1990, L'Expérience concentrationnaire. Essai sur le maintien de l'identité sociale, Paris, Métailié.

Radstone (Susannah) (Ed.), 2010, Memory: Histories, Theories, Debates, Fordham University Press.

Radstone (Susannah), 2008, «Memory studies : For and against », Memory Studies, 1, p. 31-39.

Revista Anthropos, 2008, "Maurice Halbwachs. La memoria collectiva, une categoria innovada de la sociologia actual ».

ReVue D'Histoire DeS SCIENCES HUMAINES, 2005, «Discipliner la ville. L'émergence des savoirs urbains $», 1 / 12$.

RevUe D'Histoire Des SCIENCES HUMAINes, 1999, « Maurice Halbwachs et les sciences humaines de son temps ».

Roediger (Henry) et James WerTSCH, 2008, «Creating a new discipline of memory studies », Memory studies, 1(1), p. 9-22.

Rossington (Michael) et Anne Whitehead (Eds.), 2007, Theories of Memory. A reader, Crawley, W.A., University of Western Australia Press.

Rousso (Henry), 1991, "Pour une histoire de la mémoire collective : L'après-Vichy », Cahiers de l'IHTP, 18, p. 264-278.

SABOURIN (Paul), 1997, «Perspective sur la mémoire sociale de Maurice Halbwachs », Sociologie et sociétés, XXIX(2), p. 139-161.

SAITO (Hiro), 2006, «Reiterated Commemoration: Hiroshima as National Trauma», Sociological Theory, 24(4), p. 353-376.

SCHWARTZ (Barry), 1996, « Memory as a Cutlural System : Abraham Lincoln in World War II », American Sociological Review, 61(5), p. 908-927.

SCHWARTZ (Barry), 1991, « Social change and collective memory: the Democratization of Georges Washington », American Sociological Review, 56, p. 221-236. 
SChWARTz (Barry), 1982, «The Social Context of Commemoration : A Study in Collective Memory », Social Forces, 61(2), p. 374-402.

SCHUMAN (Harold) et Barry SchwARTZ, 2005, «Commemoration and Belief: Abraham Lincoln in American Memory, 1945-2001», American Sociological Review, 70(2), p. 183203.

SCHUMAN (Harold) et Jacqueline SCOTT, 1989, "Generations and collective memories », American Sociological Review, 54, p. 351-381.

SpILlman (Lynn), 1998, «When Do Collective Memories Last? Founding Moments in the United States and Australia », Social Science History, 22(4), p. 445-477.

SuTTON (John), 2009, « Looking beyond memory studies : Comparisons and integrations », Memory Studies, 2, p. 299-301.

TOPALOV (Christian), 2008, «Sociologie d'un étiquetage scientifique : urban sociology (Chicago, 1925) », L’Année sociologique, 58 (1), p. 203-234.

Topalov (Christian), 2006a, «Maurice Halbwachs : l'expérience de Chicago (automne 1930) », Annales. Histoire, sciences sociales, 61(3), p. 555-581.

Topalov (Christian), 2006b, « Maurice Halbwachs et les sociologues de Chicago », Revue française de sociologie, 47(3), p. 561-590.

Topalov (Christian), 2004, «Les usages stratégiques de l'histoire des disciplines. Le cas de l'« école de Chicago » en sociologie », dans HeILBRon (Johan), Remi LenOIR et Gisèle SAPIRO (eds.), Pour une histoire des sciences sociales. Hommage à Pierre Bourdieu, Paris, Fayard, p. 127-157.

TOPALOV (Christian), 1999, « «Experiences sociologiques » : les faits et les preuves dans les thèses de Maurice Halbwachs (1909-1913) », Revue d'histoire des sciences humaines, 1, p. $11-46$.

VAN DAMME (Stéphane), 2005, «Les sciences humaines à l'épreuve de la ville : les enjeux d'une archéologie des savoirs urbains (XVII-XX siècle)», Revue d'histoire des sciences humaines, 12, p. 3-15.

VERRET (Michel), 1972, «Halbwachs ou le deuxième âge du durkheimisme », Cahiers internationaux de sociologie, III, p. 311-336.

VINITZKY-SEROUSSI (Vered), 2002, «Commemorating a Difficult Past: Yitzhak Rabin's Memorials », American Sociological Review, 67, p. 30-51.

WetZEL (Dietmar J.), 2009, Maurice Halbwachs, Uvk Verlag Gmbh.

WinTER (Jay), 2000, «The Generation of Memory: Reflections on the Memory Boom in Contemporary Historical Studies », Bulletin of the German Historical Institute, 27, p. 69-92. 
WinTER (Jay), 2006, « Notes on the Memory Boom : War, Remembrance, and the Uses of the Past », dans Bell (Duncan) (Ed.), Memory, trauma and world politics : reflections on the relationship between past and present, New York, Palgrave Macmillan.

WinTER (Jay) et Emmanuel SivAn, 1999, War and remembrance in the Twentieth Century, Cambridge, Cambridge University Press.

Woods (Harriet) et Antonia Susan ByatT, 2008, Memory, London, Chatto \& Windus.

Wuthnow (Richard), 1987, Meaning and Moral Order: Explorations in Cultural Analysis, Berkeley and Los Angeles, University of California Press.

ZONABEND (Françoise), 1980, La mémoire longue. Temps et histoire au village, Paris, PUF. 


\section{Annexe}

Recherche sur Google (effectuée au 1510 2010)

\begin{tabular}{|l|l|l|}
\hline Premier mot clef & Second mot clef & Nombre d'entrée trouvées \\
\hline « Memory studies » & Néant & 57600 \\
\hline « Memory studies » & Halbwachs & 3210 \\
\hline « Memory studies » & Nora & 3200 \\
\hline « Memory studies » & Olick & 2790 \\
\hline « Memory studies » & Bartlett & 2400 \\
\hline « Memory studies » & Assmann & 1880 \\
\hline « Memory studies » & Hutton & 1060 \\
\hline « Memory studies » & Schwatrz (Barry) & 855 \\
\hline « Memory studies » & Winter (Jay) & 695 \\
\hline « Memory studies » & Warburg & 503 \\
\hline
\end{tabular}

Recherche sur Google Scholar (effectuée au 1510 2010)

\begin{tabular}{|l|l|l|}
\hline Premier mot clef & Second mot clef & Nombre d'entrée trouvées \\
\hline « Memory studies » & Néant & 9840 \\
\hline « Memory studies » & Halbwachs & 927 \\
\hline « Memory studies » & Nora & 791 \\
\hline « Memory studies » & Olick & 631 \\
\hline « Memory studies » & Bartlett & 676 \\
\hline « Memory studies » & Assmann & 330 \\
\hline « Memory studies » & Schwatrz (Barry) & 279 \\
\hline « Memory studies » & Winter (Jay) & 260 \\
\hline « Memory studies » & Hutton & 253 \\
\hline « Memory studies » & Warburg & 94 \\
\hline
\end{tabular}


Recherches sur JSTOR (effectuée au 1510 2010)

\begin{tabular}{|l|l|l|l|}
\hline Premier mot clef & Second mot clef & Intervalle & Nombre d'entrées trouvées \\
\hline « Memory studies » & Néant & $2007-2010$ & 65 \\
\hline « Memory studies » & Olick & $2007-2010$ & 21 \\
\hline « Memory studies » & Halbwachs & $2007-2010$ & 18 \\
\hline « Memory studies » & Schwartz (Barry) & $2007-2010$ & 15 \\
\hline « Memory studies » & Nora & $2007-2010$ & 13 \\
\hline « Memory studies » & Assmann & $2007-2010$ & 8 \\
\hline « Memory studies » & Bartlett & $2007-2010$ & 7 \\
\hline « Memory studies » & Winter & $2007-2010$ & 5 \\
\hline « Memory studies » & Hutton & $2007-2010$ & 3 \\
\hline « Memory studies » & Warburg & $2007-2010$ & 2 \\
\hline
\end{tabular}

\begin{tabular}{|l|l|l|l|}
\hline Premier mot clef & Second mot clef & Intervalle & Nombre d'entrées trouvées \\
\hline « Memory studies » & Néant & Sans & 489 \\
\hline « Memory studies » & Halbwachs & Sans & 83 \\
\hline « Memory studies » & Nora & Sans & 80 \\
\hline « Memory studies » & Olick & Sans & 70 \\
\hline « Memory studies » & Schwartz (Barry) & Sans & 67 \\
\hline « Memory studies » & Assmann & Sans & 19 \\
\hline « Memory studies » & Bartlett & Sans & 29 \\
\hline « Memory studies » & Winter & Sans & 33 \\
\hline « Memory studies » & Hutton & Sans & 27 \\
\hline « Memory studies » & Warburg & Sans & 22 \\
\hline
\end{tabular}


Notice biographique

Née en 1976, Sarah Gensburger est chargée de recherche au CNRS (Institut de Sciences Sociales du Politique). Elle étudie la constitution de la «mémoire» en question sociale contemporaine. Elle a notamment publié Les «'Justes' de France ». Politiques publiques de la mémoire (Presses de Sciences Po, 2010) et «Fragments de mémoire collective : les Justes parmi les Nations » in M. Halbwachs, La Topographie légendaire des évangiles en terre sainte, réédition dirigée par Marie Jaisson (PUF, 2008). 